\title{
A voice-activated key for the Apple Macintosh computer
}

\author{
SALVADOR ALGARABEL and JAIME SANMARTIN \\ University of Valencia, Valencia, Spain \\ and \\ FRANCISCO AHUIR \\ Sateco Ordenadores, S.L., Valencia, Spain
}

An interface circuit to connect a microphone to an Apple Macintosh computer is described. The Apple Macintosh mouse port is used as the input port, and the microphone activation simulates a mouse press.

One of the main drawbacks of the Macintosh Computer is its closed architecture, particularly with the $512 \mathrm{~K}$ and the 1-MB versions, which do not have expansion slots. There are some systems (Chute, Gaeman, \& Ziegler, 1987; Reed College, 1985) that break this closeness and offer a number of versatile possibilities at a high cost. We designed a simple circuit that uses the Macintosh input devices to enable a microphone to be connected to the computer. Although the circuit is not as versatile as some of the commercial, general purpose interfaces, it can be used to run experiments at low cost.

\section{Circuit Description}

In the design, we use the mouse input: that is, the serial RS232/RS242 port is used as an input port, and the circuit is designed so that a pulse coming from the microphone is interpreted as a mouse press by the computer hardware. In this arrangement, the interface is connected to the mouse port, and the mouse and the microphone are both connected to the interface circuit. Figure 1 shows an external diagram of the interface housing. An adjustable sensitivity dial allows the user to establish a reference level for triggering the circuit. Two additional on/off switches, one for the power supply and the other for the microphone connections, allow the circuit to be permanently connected, sometimes using the microphone, and at other times the mouse itself. Three diodes indicate when the interface is ready or activated.

Figure 2 is a diagram of the circuit, which is built around four operational amplifiers. The first amplifier is set in a noninverting configuration to attain a high input impedance and, with a gain of a factor of about 100 , to set the signal at a usable level. A potentiometer is in the circuit to vary the sensitivity level. A second amplifier

This research was supported by Grant PB86-0311 from The Dirección General de Investigación Científica y Técnica del Ministerio de Educación y Ciencia. Requests for reprints should be sent to Salvador Algarabel, Departmento de Psicologia Experimental, Universidad de Valencia, Blasco Ibánez 21, 46010 Valencia, Spain.
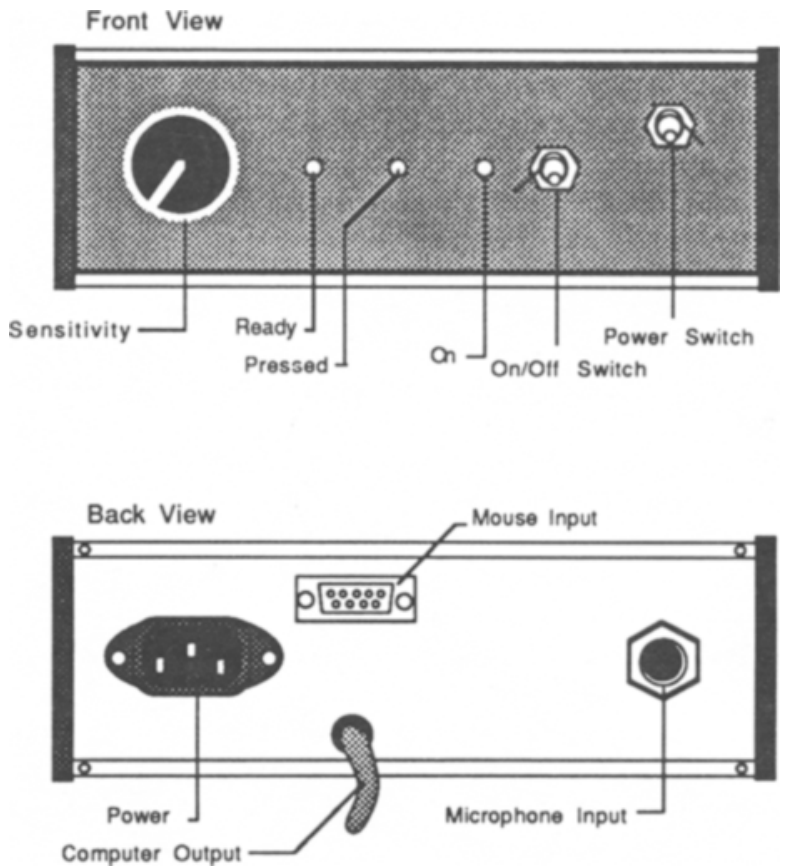

Figure 1. Views of the interface box housing the circuit.

is set in an inverting configuration with a gain factor of 10. The third operational amplifier is set as a comparator. A variable resistor establishes the voltage reference level so that if the voltage of the other input goes above the reference level, the amplifier output goes positive, and this output is used to simulate the mouse press.

\section{Test}

To check the validity of the circuit, we wrote a program to test the use of the microphone in a naming experiment in which reaction time is measured when a subject sees two stimuli in sequence and must name the second one (Balota \& Chumbley, 1984). The voice is de- 


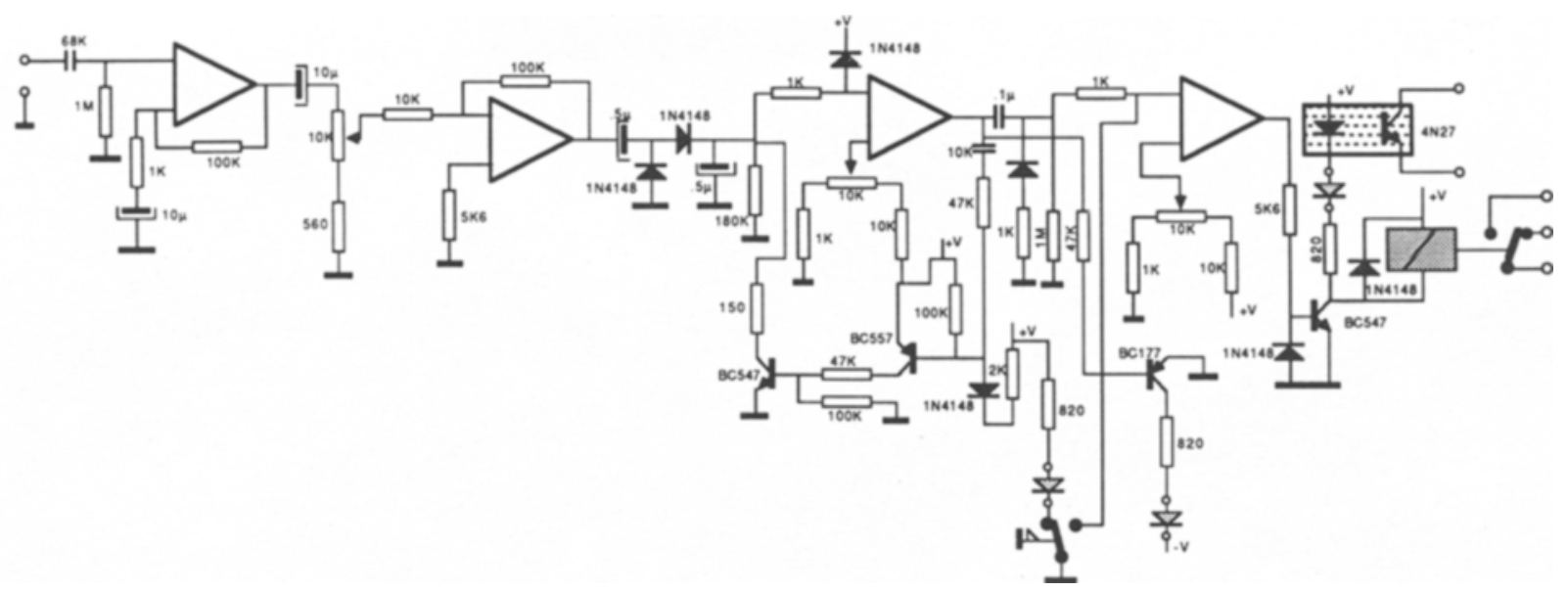

Figure 2. Circuit diagram of the microphone interface to the Apple Macintosh.

tected simply as a mouse press. To make the process more understandable, we wrote a short program in Microsoft BASIC 3.0 to implement a real experiment, showing in this manner the use of the circuit.

The experiment consisted of 63 trials, in which a prime word was followed by a prime target that the subject had to pronounce. Twenty-one of those trials contained related pairs of words extracted from association norms. The remaining 42 trials comprised 21 control trials in which the prime word was always the word "NEUTRO" ("neutral"), and 21 in which the prime and the target were unrelated pairs of words. We used a stimulus onset asynchrony between prime and target of $250 \mathrm{msec}$, with a prime duration of $200 \mathrm{msec}$.

The appendix presents a listing of the program written to run the experiment. We used two previously published routine libraries: one for accurate stimulus exposure (Lane \& Ashby, 1987), and the other to measure naming time (Westall, Perkey, \& Chute, 1986). The listing is written in a modular manner and, after array definitions and main program specification are presented, the main experimental functions are included as subroutines. Of particular interest is the instruction "WHILE PEEK $(\& H 172)<>0$ : WEND," which checks whether the mouse has been pressed, or detects when the interface is connected. It is important to realize that PEEKing directly position \&H172 avoids any false triggering of the circuit due to the Macintosh buffer that accumulates all mouse events. The same function can be accomplished with the following instructions: "WHILE MOUSE(0)>-1:WEND." The use of this last instruction requires that the mouse event buffer be discharged during the intertrial interval; otherwise, the program would be triggered by spurious activations produced during the intertrial interval.

The results of our experiment parallel the results obtained by other investigators, and are within a range of values similar to others collected with different hardware.

\section{REFERENCES}

Balota, D. A., \& Chumbley, J. I. (1984). Are lexical decisions a good measure of lexical access? The role of word frequency in the neglected decision stage. Journal of Experimental Psychology: Learning, Memory, \& Cognition, 10, 340-357.

Chute, D. L., Gaeman, D., \& Ziegler, T. (1987). Maclaboratory Controller: A switch and A/D interface between Apple's Macintosh and peripheral apparatus. Behavior Research Methods, Instruments, \& Computers, 19, 205-209.

LANe, D. M., \& Ashry, A. B. (1987). Psychlib: A library of machine language routines for controlling psychological experiments on the Apple Macintosh computer. Behavior Research Methods, Instruments, \& Computers, 19, 246-248.

REED COLLEGE. (1985). Rascal user manual: Macintosh language for real time $1 / O$ oriented development. Portland, OR: Metaresearch.

Sanmartin, J. (1986). Subntinas para generar secuencias pseudoaleatorias simples. Psicologica, 7, 91-98.

Westall, R., Perkey, M. N., \& Chute, D. L. (1986). Accurate millisecond timing on Apple's Macintosh using Drexel's MilliTimer. Behavior Research Methods, Instruments, \& Computers, 18, 307-311.

APPENDIX

Program Listing for Naming Demonstration Program

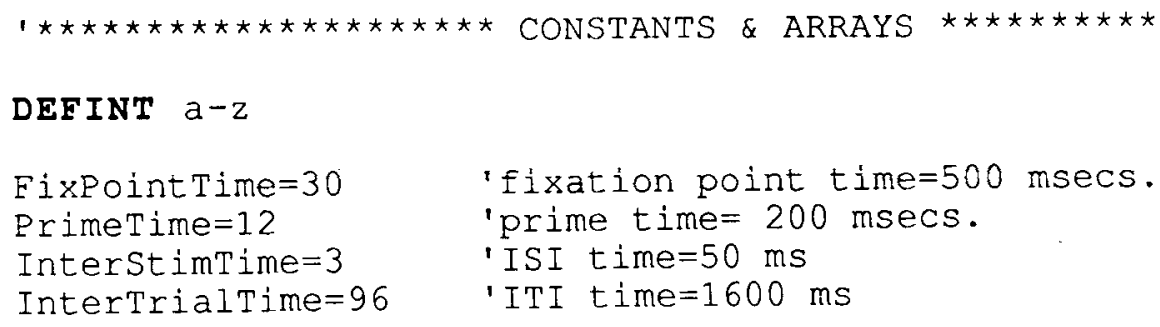

FixPoint $\mathrm{Time}=30$

PrimeTime $=12$

InterstimTime $=3$

InterTrialtime $=96$

'fixation point time $=500$ msecs.

'prime time $=200$ msecs.

' ISI time $=50 \mathrm{~ms}$

- ITI time $=1600 \mathrm{~ms}$ 
NTrials $=63$

NSessions $=1$

Hpoint $=220$

Vpoint $=150$

maxWordLen $=12$

True $=1$

False $=0$

MyTime $=0$

NewTime $=0$

'number of trials

'number of sessions

'horizontal coordinates for stimulus presentation

'vertical coordinates for stimulus presentation

'maximum length for stimulus words

'value needed to install the millicontrol routine

'value to deinstalled the millicontrol routine

'starting time to measure naming

'final naming time

DIM CountCode (11)

DIM ControlCode (85)

DIM primes (NTrials, NSessions)

DIM target\$(NTrials, NSessions)

DIM condition (NTrials, NSessions)

DIM TR(NTrials, NSessions)
'Millicount array

'MilliControl array

'prime stimuli

'target stimuli

'experimental condition codes

'naming times
RANDOMIZE TIMER

LIBRARY "PSYchLib"

GOSUB InstallRoutines

GosuB Subjet Information

StimFile $\$=$ FILES $\$$ (1, "TEXT")

GosuB ReadStimEile

GOSUB Preparescreen

GOSUB Randomsecuence

GOSUB Initmillitimer

FOR session=1 TO NSessions

GOSUB Initsession

FOR trial=1 TO NTrials

GosuB Exectrial

NEXT trial

NEXT session

GOSUB DiscardMillitimer

GosuB FinalMessage

GOSUB SaveResponses

END

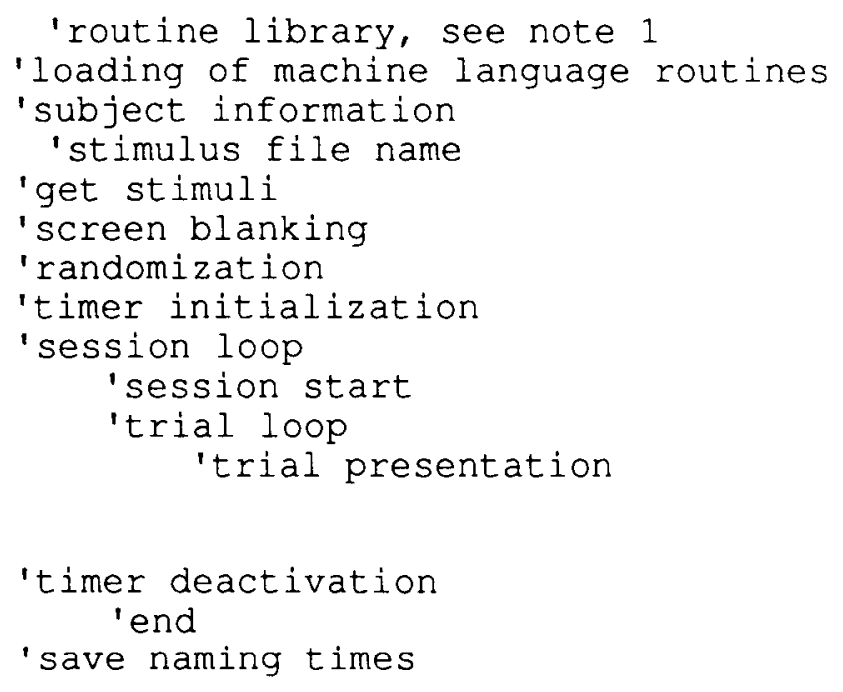

SUBROUTINES

InstallRoutines:

FOR KOUNT=0 TO 10

'see note 2

RmAD Count Code (KOUNT)

NEXT KOUNT

FOR KOUNT $=0$ TO 84

READ Controlcode (KOUNT)

RETURN

NEXT KOUNT

Subjet Information:

INPUT" CODE:"; SubjetCode\$

INPUT" NAME:"; SubjetName\$

INPUT" AGE:"; SubjetAge\$

CLS

RETURN 


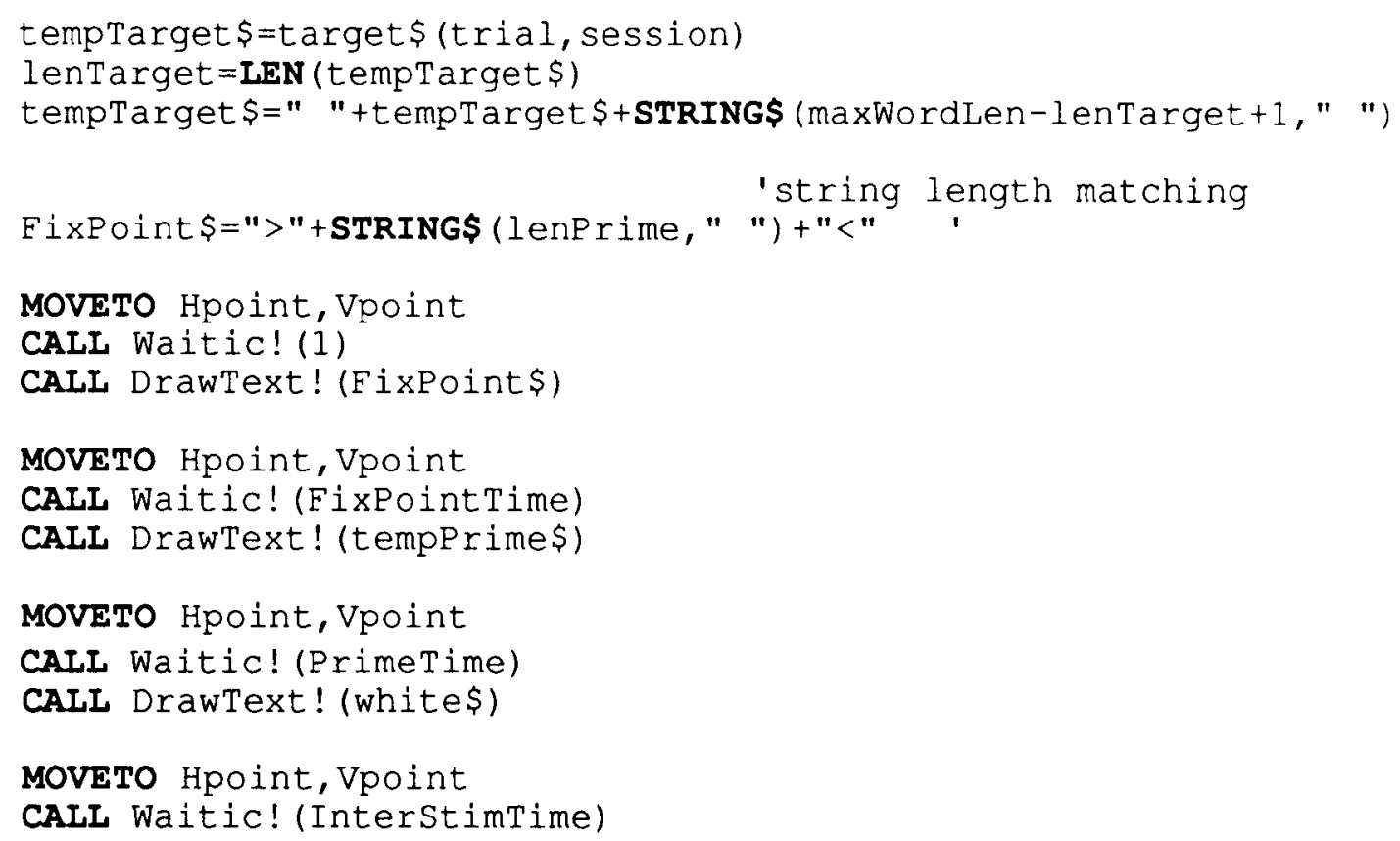

CALL Millicount!(VARPTR(NewTime)) 'naming time

\section{CLS}

$\operatorname{TR}($ trial, session) $=$ NewTime-MyTime-9 'this is the final naming time version)

' corrected for compile

execution

'If the program is written with the interpreter, the

'final time must be corrected by $17 \mathrm{~ms}$. which is

'the error introduced by the interpreter in command

\section{RETURN}

CALL Waitic!(InterTrialTime)

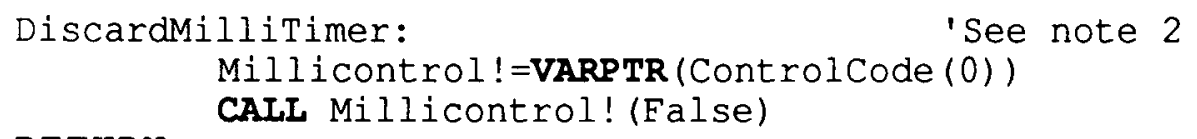

Einalmessage:

WHILE INKEY\$<>" ":WEND

PRINT "This is the end of the experiment" 


\section{PRINT "Thank you for your collaboration" \\ PRINT "Press the spacebar to end" \\ WHILE INKEY\$<>" ":WEND INITCURSOR \\ RETURN}

\section{SaveResponses:}

FileName $\$=$ St imF ile $\$+$ Subjet Code $\$$

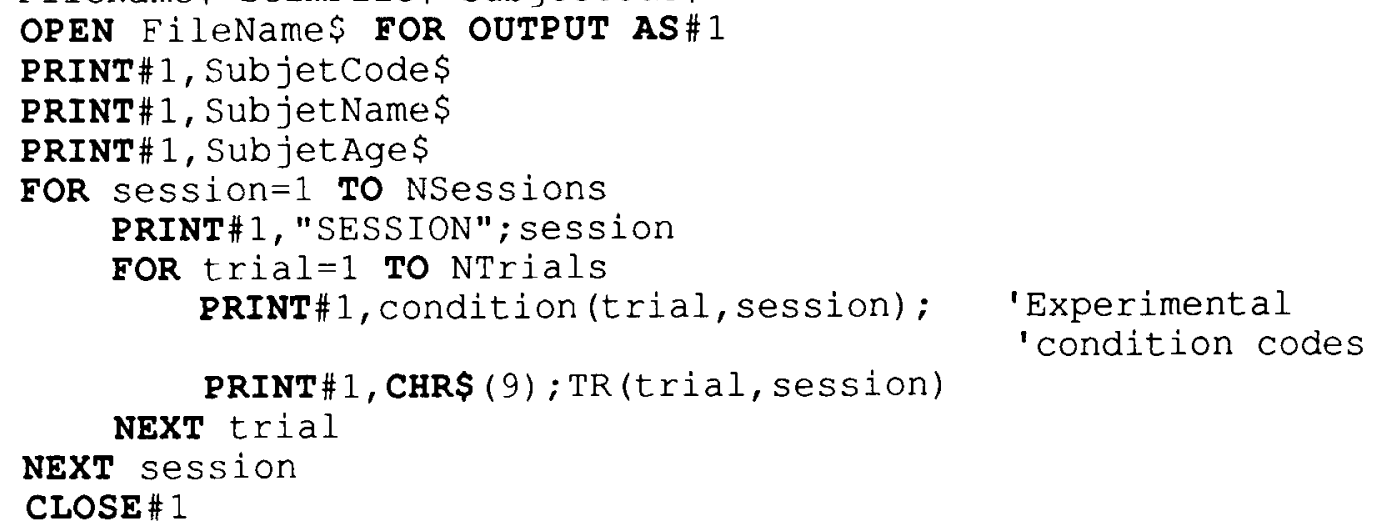

1. Routines BLS!, Waitic! (), and DrawText! () are part of the PsychLib library (Lane \& Ashby, 1987). This library is required to be able to use those instructions.

2. Routines MilliControl and MilliCount were adapted from Westall, Perkey, and Chute (1986).

3. Adaptation from routines presented in Sanmartin (1986). 